\title{
Controle remoto ou remoto controle? A economia comportamental e a promoção de comportamentos saudáveis
}

\author{
Mathias Roberto Loch, ${ }^{1}$ Douglas Fernando Dias, ${ }^{1}$ \\ Alex Sandro Rodrigues Castro ${ }^{2}$ e Paulo Henrique Guerra ${ }^{3}$
}

Como citar Loch MR, Dias DF, Castro ASR, Guerra PH. Controle remoto ou remoto controle? A economia comportamental e a promoção de comportamentos saudáveis. Rev Panam Salud Publica. 2019;43:e18. https://doi.org/10.26633/RPSP.2019.18

RESUMO A economia comportamental é um campo de saberes e práticas que estuda o efeito de fatores psicológicos, cognitivos, emocionais e sociais sobre a tomada de decisão de indivíduos e instituições. Essa disciplina parte da premissa de que as escolhas humanas não são resultado de uma cuidadosa ponderação de custos e benefícios e de que alguns aspectos (por exemplo, opção padrão, enquadramento e efeito-âncora) podem influenciar a tomada de decisão por parte das pessoas. Assim, o objetivo deste texto é apresentar aspectos gerais da economia comportamental e reflexões sobre sua possível aplicação na promoção de comportamentos saudáveis. Uma das funções dos profissionais de saúde é fazer com que a adoção de comportamentos saudáveis seja mais fácil por parte das pessoas. Nessa perspectiva, existem evidências de que a aplicação de ideias da economia comportamental pode ter impacto positivo na adoção de comportamentos de saúde diversos, como, por exemplo, escolhas alimentares, prática de atividade física, adesão a campanhas de vacinação e doação de órgãos. Desse modo, acredita-se que a apropriação de alguns conceitos e insights da economia comportamental possa, de um lado, promover comportamentos saudáveis e, de outro, apoiar ações mais efetivas de incentivo a esses comportamentos.

Palavras-chave Economia comportamental; promoção da saúde; estilo de vida; educação em saúde.

A promoção de comportamentos saudáveis, cuja adoção é influenciada por aspectos econômicos, sociais e psicológicos, entre outros, tem sido mundialmente apontada como importante desafio para

\footnotetext{
Universidade Estadual de Londrina, Programa de Pós-Graduação em Saúde Coletiva, Londrina (PR), Brasil

2 Universidade Estadual de Campinas, Programa de Pós-Graduação em Economia, Campinas (SP), Brasil.

3 Universidade Federal da Fronteira Sul, Chapecó (SC), Brasil. Correspondência: paulo.guerra@uffs. edu.br
}

as políticas públicas. Nesse cenário, é fundamental que os profissionais envolvidos com essa temática, que por natureza é intersetorial, se apropriem de elementos que possam ajudá-los a desenvolver ações mais efetivas. Assim, as diversas teorias e modelos que buscam uma melhor compreensão do comportamento humano (1) podem ser de grande importância.

Uma abordagem emergente na área da saúde é a economia comportamental (2-4) - um campo de saberes e práticas que estuda o efeito de fatores psicológicos, cognitivos, emocionais e sociais sobre a tomada de decisão de indivíduos e instituições. Em uma busca preliminar realizada no repositório Pubmed, em 26 de abril de 2018, foram identificados 732 trabalhos que continham a expressão behavioral economics em seus títulos ou resumos, a maioria publicada a partir de 2015. O cruzamento dessa expressão com outros descritores selecionados revelou 112 resultados: 34 para food, 25 para tobacco, 25 para physical activity e oito para vaccine. A literatura mostra também que a 
aplicação de algumas ideias da economia comportamental pode influenciar escolhas em comportamentos diversos, como, por exemplo, escolhas alimentares $(5,6)$, prática de atividades físicas (7) e adesão a campanhas de vacinação (8) e de doação de órgãos (9).

Dado este cenário, o presente texto tem como objetivo apresentar alguns aspectos gerais da economia comportamental e propor reflexões iniciais sobre sua possível aplicação na promoção de comportamentos saudáveis.

\section{ASPECTOS GERAIS DA ECONOMIA COMPORTAMENTAL}

A economia comportamental se baseia na incorporação, pela economia, de descobertas e desenvolvimentos teóricos na área da psicologia e outras ciências humanas e sociais, além da neurociência. É crítica à abordagem econômica tradicional, fortemente embasada no princípio de que o ser humano é um tomador de decisões racional, ponderado e com capacidade ilimitada de processar informações $(10,11)$.

Na lógica da economia comportamental, as pessoas tomam suas decisões com base em hábitos, experiências pessoais e regras práticas simplificadas. Ao mesmo tempo em que aceitam soluções apenas satisfatórias, tomam decisões rapidamente, têm dificuldade em conciliar interesses de curto e longo prazo e são fortemente influenciadas por fatores emocionais e pelas decisões daqueles que interagem com elas $(10,11)$. Essa tomada de decisão pode também ser afetada por diversos detalhes do contexto, como, por exemplo, a opção padrão, o efeito de enquadramento e o efeitoâncora - conceitos que serão abordados mais detalhadamente no tópico do artigo que trata da possível aplicação da economia comportamental na área da saúde.

Recentemente, a economia comportamental teve importante destaque na mídia. Em 2017, o Prêmio Nobel de Economia foi dado a Richard Thaler, considerado um dos fundadores dessa área. Antes dele, Daniel Kahneman, doutor em psicologia, recebera o Prêmio Nobel (2002) por seu trabalho sobre racionalidade limitada. Entre as produções de Kahneman está o livro "Rápido e devagar: duas formas de pensar" (12), considerado um marco importante para a divulgação da economia comportamental. Nessa obra, são apresentados elementos do funcionamento da mente humana a partir especialmente da psicologia cognitiva e social. Segundo o autor, existem dois sistemas na mente humana, que ele denomina "sistema 1" e "sistema 2". Enquanto o sistema 1 toma decisões rápidas, de maneira intuitiva e sem esforço, baseadas em nossa memória associativa (a forma rápida de pensar), o sistema 2 , mais lento, é acionado quando nos encontramos em uma situação que exija concentração e raciocínio laborioso (é a forma "devagar" de pensar). $\mathrm{O}$ autor destaca que o sistema 1, rápido e intuitivo, influencia mais as nossas escolhas do que costumamos imaginar. Ademais, ao longo das últimas décadas, diversas pesquisas apontaram um impressionante catálogo de falhas de raciocínio que vêm embutidas nessa "forma rápida" de pensar.

Por isso, os economistas comportamentais costumam argumentar que os seres humanos não são agentes racionais que fazem sempre (ou pelo menos quase sempre) as escolhas que mais os beneficiam. Um aspecto importante é que as campanhas educativas parecem ter um impacto limitado na "correção" desses vieses. O problema, dessa forma, não reside apenas no fato de as pessoas não serem capazes de saberem o que querem. Pensamos que a maioria delas não tem dúvida em relação aos seus objetivos mais ou menos universais, como manter a saúde, guardar dinheiro para a compra de bens mais caros ou para a aposentadoria, por exemplo. A questão é que os vieses cognitivos são especialmente eficazes em solapar suas estratégias para chegar a esses fins. Por mais que tentemos evitar, nossos cérebros valorizam muito mais o presente do que o futuro e julgam qualquer perda como duas vezes pior do que um ganho no mesmo valor.

Outro livro considerado fundamental na popularização da economia comportamental é "Nudge: o empurrão para a escolha certa", escrito por Thaler e Sunstein (13). Esses autores defendem que, em muitos casos, as pessoas tomam decisões inferiores em termos de seu próprio bem-estar - decisões essas que mudariam se o tomador de decisão tivesse informações completas, habilidades cognitivas ilimitadas e total autocontrole. Os autores sugerem ainda que os dados disponíveis sobre obesidade, hábito de fumar e ingestão de bebida alcoólica demonstram que as pessoas não tomam decisões que são de seu interesse o tempo todo (nem na maior parte do tempo). Também sugerem o quanto mudanças aparentemente pequenas na forma como os sistemas funcionam podem ajudar as pessoas a exercerem melhor o autocontrole, por exemplo, ao escolher o que comer na cantina da escola ou do trabalho. $\mathrm{Na}$ visão dos autores, é possível criar condições (um "empurrãozinho", do inglês nudge) para que as pessoas façam, em diferentes áreas de vida, escolhas mais "adequadas".

O conceito de nudge é elaborado pelos autores dentro de um "paternalismo libertário", considerado como ponto intermediário entre o total liberalismo (entendido como nenhuma intervenção do Estado na vida privada das pessoas) e o paternalismo coercitivo (entendido como o Estado tomando todas as decisões em nome das pessoas). Nesse conceito, é desejável manter a possibilidade de escolha, mas seria também desejável que o Estado incentivasse as escolhas consideradas socialmente corretas ou saudáveis. Em outras palavras, Thaler e Sunstein acreditam que o Estado pode e deve induzir o cidadão a optar pelo que se afigura como a melhor decisão, sem, todavia, obrigá-lo a isso. É preciso, defendem eles, sempre deixar uma porta de saída, para que situações particulares possam receber soluções particulares. Evidentemente, essa discussão é complexa e transcende os objetivos deste texto.

"Previsivelmente irracional: como as situações do dia-a-dia influenciam as nossas decisões" (14), de Dan Ariely, é outra publicação de impacto na divulgação da economia comportamental e mostra, a partir da apresentação de uma série de experimentos, o quanto a nossa "irracionalidade" nas decisões é previsível. A previsibilidade se relaciona com o fato de que certos erros de decisão que cometemos são sistemáticos, e não aleatórios. Ou seja: cometemos frequentemente os mesmos "erros". Ariely argumenta que, sabendo como os repetimos vez após vez, poderemos começar a perceber como evitá-los.

Muitas outras produções poderiam ser mencionadas. No contexto brasileiro, destacamos o guia organizado por Ávila e Bianchi, publicado em 2015 (10), com textos de pesquisadores brasileiros e internacionais. 
Em síntese, são pontos importantes sobre a economia comportamental:

- certamente não é a "invenção da roda", já que há décadas existem teorias e modelos que buscam um melhor entendimento do comportamento humano;

- as pesquisas realizadas na economia comportamental abordam e podem ser aplicadas em distintas temáticas, inclusive na área da saúde, indo muito além do que boa parte das pessoas imagina como sendo o objeto da economia mais tradicional;

- um princípio fundamental é que nossas "escolhas" são menos baseadas na razão do que se costuma imaginar - nossas decisões não são resultado de uma cuidadosa ponderação entre custos e benefícios e muitas vezes não se baseiam em preferências preexistentes;

- qualquer detalhe (alguns que sequer são percebidos) pode fazer grande diferença nas tomadas de decisões - a estratégia comum de intervenção da economia comportamental consiste em alterar esses detalhes do contexto de decisão de modo a induzir os agentes a fazerem escolhas mais "acertadas";

- finalmente, apesar de nossas escolhas não seguirem, em boa medida, uma lógica "racional", elas não são aleatórias, sendo possível, em muitos casos, prever como serão.

\section{APLICAÇÃO DA ECONOMIA COMPORTAMENTAL NA PROMOÇÃO DE COMPORTAMENTOS SAUDÁVEIS: ENTRE A CAUTELA E A POTENCIALIDADE}

Apesar de reconhecermos que a informação é necessária na práxis em saúde (por exemplo, em atividades de aconselhamento e grupos de educação em saúde, entre outros), e que deve ser função dos profissionais a divulgação de informações válidas a respeito da saúde de um modo geral, muitas vezes essa abordagem, focada somente na informação, é limitada, pois baseia-se, via de regra, em modelos parciais do comportamento humano, que negligenciam ou subestimam o papel dos processos não conscientes que determinam nossas escolhas (2). Complementarmente, é importante dizer que muitas vezes, na área da saúde, a essa visão simplista soma-se uma visão moralista, que determina como "certos" ou "errados" os comportamentos relacionados à saúde (15), desconsiderando uma série de fatores sociais e econômicos das pessoas. Acreditamos que uma das funções dos profissionais de saúde é fazer com que a adoção de comportamentos saudáveis seja mais fácil por parte das pessoas, sem julgamento moral de comportamentos.

A abordagem centrada na informação muitas vezes é adotada como solução única pelos governos, talvez pela conveniência de evitar possíveis conflitos com setores que têm forte influência econômica e política (como a indústria de alimentos ultraprocessados). Por outro lado, alguns países (como Reino Unido, Austrália, Alemanha) formaram equipes de especialistas em ciência comportamental destinadas a delinear ações com potencial de encorajar comportamentos saudáveis, sem restringir as opções de escolha (3).

Um conceito relevante da economia comportamental é o de alteração da opção padrão. Esse conceito pode se aproximar da área da saúde, por exemplo, no momento da definição da posição dos alimentos em cantinas, mercearias ou lanchonetes. Tendo em vista que a posição dos alimentos nas prateleiras pode afetar a probabilidade de que sejam escolhidos, uma ação baseada na economia comportamental seria a de colocar os alimentos considerados mais saudáveis na posição principal. Essa estratégia não remove as opções menos saudáveis, mas as transfere para locais menos privilegiados, que nossos hábitos ajudam a contornar (4).

Também é importante o efeito de enquadramento, que diz respeito à forma como a informação é apresentada às pessoas. Escolhas logicamente equivalentes podem ser apresentadas (enquadradas) de modo a ressaltar aspectos positivos ou negativos da mesma decisão, levando a mudanças em sua atratividade relativa. Por exemplo, descrever um hambúrguer como sendo $75 \%$ livre de gordura ou como tendo $25 \%$ de gordura transmite a mesma informação sobre o hambúrguer, mas enfatizar o aspecto "75\% livre de gordura" faz a carne ser avaliada mais favoravelmente pelas pessoas (16). Similarmente, as pessoas são mais propensas a concordar com um procedimento médico associado a uma probabilidade de salvar 90 em 100 vidas do que com um procedimento associado a um risco de perder 10 em 100 vidas (17).

Os julgamentos das pessoas também costumam ser muito influenciados pela "primeira impressão". Ou seja, as pessoas tomam a parte da informação que recebem primeiro como base ("âncora") para atribuir valores às coisas. Por exemplo, uma pessoa entra em uma loja e vê uma camisa: observa o preço na etiqueta, de $\mathrm{R} \$ 300,00$. O vendedor aborda a pessoa e diz que a camisa agora custa apenas R\$ 100,00. Talvez o indivíduo nem precise da camisa, mas um desconto de $\mathrm{R} \$ 200,00$ lhe parece realmente uma grande vantagem. O preço da etiqueta funcionou como uma âncora. $\mathrm{O}$ processo de ancoragem tem sido observado em diversos domínios e tarefas, inclusive em decisões sobre metas pessoais. Metas ambiciosas (em esportes, dieta, entre outros) como ponto de partida podem induzir respostas mais intensas por parte das pessoas (12).

Mesmo com elementos promissores, é necessário ter cautela para não superestimar o potencial da economia comportamental na saúde nem desmerecer ou ignorar outras teorias e modelos. Ademais, existem críticas à economia comportamental que precisam ser consideradas. Alguns autores argumentam que pode haver uma "racionalidade" por trás dos vieses do comportamento humano (18). De qualquer modo, destacamos a importância de uma formação mais sólida sobre essas teorias e modelos nos cursos de graduação e pós-graduação da área da saúde. A apropriação desses saberes, assim como a possibilidade de sua inserção na práxis, é potencialmente rica. É fundamental que a área da saúde incorpore, tanto na sua prática profissional, quanto nas suas pesquisas, a ideia de que o conhecimento, apesar de importante, é, em geral, insuficiente para a adoção de comportamentos saudáveis. Nesse sentido, a economia comportamental pode ser de grande utilidade.

Conflitos de interesse. Nada declarado pelos autores.

Declaração. As opiniões expressas no manuscrito são de responsabilidade exclusiva dos autores e não refletem necessariamente a opinião ou política da RPSP/PAJPH ou da Organização PanAmericana da Saúde (OPAS). 


\section{REFERÊNCIAS}

1. Glanz K, Rimer BK, Viswanath K. Health behavior and health education: theory, research, and practice. $4^{\mathrm{a}}$ ed. São Francisco: Jossey-Bass; 2008.

2. Marteau TM. Changing minds about changing behaviour. Lancet. 2018;391(10116): 116-7.

3. Benartzi S, Beshears J, Milkman KL, Sunstein CR, Thaler RH, Shankar M, et al. Should governments invest more in nudging? Psychol Sci. 2017;28(8): 1041-55.

4. Volpp KG, Asch DA. Make the healthy choice the easy choice: using behavioral economics to advance a culture of health. QJM. 2017;110(5):271-5.

5. Loeb KL, Radnictz C, Keller KL, Schwartz MB, Zucker N, Marcus S, et al. The application of optimal defaults to improve elementary school lunch selections: proof of concept. J Sch Health. 2018;88(4):265-71.

6. Carroll KA, Samek A, Zepeda L. Food bundling as a health nudge: Investigating consumer fruit and vegetable selection using behavioral economics. Appetite. 2018;121:237-48.

7. van der Swaluw K, Lambooji MS, Mathijssen JJP, Schipper M, Zeelenberg M,
Berkhout S, et al. Physical activity after commitment lotteries: examining longterm results in a cluster randomized trial. J Behav Med. 2018;41(4):483-93.

8. Yagi A, Ueda Y, Kimura T. A behavioral economics approach to the failed HPV vaccination program in Japan. Vaccine. 2017;35(50):6931-33.

9. van Dalen HP, Henkens K. Comparing the effects of defaults in organ donation systems. Soc Sci Med. 2014;106:137-42.

10. Avila F, Bianchi A. Guia de economia comportamental e experimental. $1^{a}$ ed. São Paulo: EconomiaComportamental.org; 2015.

11. Castro ASR. Economia comportamental: caracterização e comentários críticos [dissertação]. Campinas: Universidade Estadual de Campinas; 2014. Disponível em: https://fernandonogueiracosta.files. wordpress.com/2015/08/castro-alex-sandro-rodrigues-de-economia-comportamental.pdf Acessado em outubro de 2018.

12. Kahneman D. Rápido e devagar: duas formas de pensar. $1^{\text {a }}$ ed. Rio de Janeiro: Objetiva; 2012.

13. Thaler R, Sunstein C. Nudge: o empurrão para a escolha certa. Rio de Janeiro: Elsevier; 2009.
14. Ariely D. Previsivelmente irracional: como as situações do dia-a-dia influenciam as nossas decisões. Rio de Janeiro: Elsevier; 2008.

15. Castiel LD, Álvarez-Dardet Diaz C. A saúde persecutória: os limites da responsabilidade. Rio de Janeiro: Editora Fiocruz; 2007.

16. Levin IP, Gaeth GJ. How consumers are affected by the framing of attribute information before and after consuming the product. J Consumer Res. 1988;15(3): 374-8.

17. Redelmeier D, Rozin P, Kahneman D. Understanding patients' decisions: Cognitive and emotional perspectives. JAMA. 1993;270(1):72-6.

18. Gigerenzer G, Selten R. Bounded rationality: the adaptive toolbox. Cambridge: MIT Press; 2001.

Manuscrito recebido em 10 de junho de 2018. Aceito em versão revisada em 5 de setembro de 2018

Behavioral economics is a field of knowledge and practices that studies the effect of psychological, cognitive, emotional, and social factors relating to decision-making by individuals and institutions. It assumes that human choices are not the result of careful consideration of costs and benefits, and that some aspects (e.g., defaults, framings,

\section{Remote control or unlikely control? Behavioral economics and the promotion of healthy behaviors}

Keywords and anchoring effects) may influence an individual's decision-making. Thus, the aim of this essay is to present some general aspects of behavioral economics and discuss its possible application in the promotion of healthy behaviors. One of the roles of health care professionals is to facilitate the adoption of healthy behaviors by the population. There is evidence of a positive influence of some behavioral economics concepts regarding the adoption of various health behaviors, such as food choices, physical activity, adherence to vaccination campaigns, and organ donation. Thus, it is believed that the appropriation of some of these concepts and insights may both promote healthier behaviors and support more effective actions to encourage these healthy behaviors.

Economics, behavioral; health promotion; life style; health education. 
RESUMEN La economía comportamental es un campo de conocimientos y prácticas que estudia el efecto de los factores psicológicos, cognoscitivos, emocionales y sociales sobre la toma de decisiones de las personas y las instituciones. Esa disciplina parte de la premisa de que las formas de elección humana no son el resultado de una cuidadosa

¿Control remoto o remota probabilidad de control? La economía comportamental y la promoción de determinados patrones de comportamiento

Palabras clave ponderación de los costos y beneficios, y de que algunos aspectos (por ejemplo, la opción predeterminada, el efecto de enmarcación y el efecto de anclaje) pueden influir en la toma de decisiones por parte de las personas. Por ende, el objetivo de este texto es presentar los aspectos generales de la economía comportamental y algunas reflexiones sobre su posible aplicación en la promoción de patrones de comportamiento saludable. Una de las funciones de los profesionales de salud es facilitar la adopción de patrones de comportamiento saludable por parte de la población. En esa perspectiva, se ha comprobado que la aplicación de los conceptos de la economía comportamental puede tener un efecto positivo en la adopción de diversos patrones de comportamiento en materia de salud, por ejemplo, la selección de alimentos, la práctica de la actividad física, la adhesión a campañas de vacunación y la donación de órganos. De ese modo, se cree que la apropiación de algunos conceptos y percepciones de la economía comportamental permite, por un lado, promover los patrones de comportamiento saludable y, por otro, apoyar medidas más eficaces para incentivar esos tipos de comportamiento.

Economía del comportamiento; promoción de la salud; estilo de vida; educación en salud. 